\title{
Constrictive pericarditis diagnosed following liver transplantation
}

\author{
Nilufer CETINER ${ }^{1}$ (D), Berna SAYLAN CEVIK ${ }^{2}$ (D), Engin TUTAR ${ }^{3}$ (D), Elif EROLU² (D), Koray AK ${ }^{4}$ (D), Figen AKALIN ${ }^{2}$ \\ ${ }^{1}$ Division of Pediatric Cardiology, Department of Child Health and Pediatrics, Faculty of Medicine, Koc University, Istanbul, Turkey. \\ ${ }^{2}$ Division of Pediatric Cardiology, Department of Child Health and Pediatrics, School of Medicine, Marmara University, Istanbul, Turkey. \\ ${ }^{3}$ Division of Pediatric Gastroenterology, Department of Child Health and Pediatrics, School of Medicine, Marmara University, Istanbul, Turkey. \\ ${ }^{4}$ Department of Cardiovascular Surgery, School of Medicine, Marmara University, Istanbul, Turkey.
}

Corresponding Author: Nilufer CETINER

E-mail: nilufercetiner@hotmail.com

Submitted: 06.12.2020 Accepted: 23.03.2021

\begin{abstract}
Constrictive pericarditis is frequently diagnosed during evaluation of the patients for hepatosplenomegaly and easily missed if not considered in differential diagnosis. Herein, we present a patient diagnosed with constrictive pericarditis during his investigation for impaired liver functions and right heart failure one year after liver transplantation.

A thirteen-year-old boy presented with abdominal distention, dyspnea and fatigue. He had undergone liver transplantation due to liver failure in the previous year, and the symptoms had recurred in the last three months. Physical examination revealed normal heart sounds. Abdominal distention and ascites were present. Cardiothoracic index and pulmonary vascular markings were normal on chest X-ray. Echocardiography showed biatrial dilatation. Thickening of the pericardium with calcifications was demonstrated by thorax computerised tomography. High pulmonary wedge pressure and equalization of end-diastolic pressures were found during catheter-angiography. Pericardiectomy was performed, histopathology was compatible with chronic fibrinous pericarditis. The patient improved dramatically after surgery, the right heart failure findings resolved, and the liver graft functions turned to be normal. Constrictive pericarditis must be considered in differential diagnosis of hepatosplenomegaly, liver dysfunction and right heart failure since surgical treatment is possible and lifesaving.

Keywords: Constrictive pericarditis, Echocardiography, Liver transplantation, Pericardiectomy
\end{abstract}

\section{INTRODUCTION}

Constrictive pericarditis (CP) is a disease presenting with manifestations of right heart failure and hepatosplenomegaly. Hepatosplenomegaly is thickening, fibrosis, adhesion and rigidity of pericardial membranes and causes impaired diastolic filling of the ventricles [1,2]. Patients with $\mathrm{CP}$ are usually diagnosed during the investigation of hepatosplenomegaly and liver disease. Diagnosis may be easily missed if CP is not considered in a differential diagnosis. Right heart failure together with massive ascites after liver transplantation (LT) is a rare condition, the causes of which may include bacterial peritonitis, obstruction of portal or hepatic veins, graft rejection, and renal or cardiac dysfunction. Identification and correction of the underlying cause is crucial [3]. It is important to consider $\mathrm{CP}$ in the etiology of liver failure, since it can be easily treated with surgical intervention. To the best of our knowledge, there has been no previous report of right heart failure accompanying massive ascites developing after LT due to CP in children. We report a case diagnosed with $\mathrm{CP}$ suggesting liver dysfunction with findings of right heart failure and massive ascites one year after LT due to diagnosis of chronic liver disease in our clinic.

\section{CASE REPORT}

A thirteen-year-old boy presented to our clinic with abdominal swelling, shortness of breath, and early fatigue. The patient had undergone LT with a diagnosis of chronic liver disease in the previous year and the pre-transplantation symptoms had recurred over in the last three months. He was referred to our clinic for evaluation for re-transplantation. In physical examination arterial blood pressure was $110 / 70 \mathrm{mmHg}$ in both arms, and heart rate was $86 / \mathrm{min}$; his skin was pale, bilateral pretibial pitting edema was present. Cardiovascular system examination revealed normal rhythmic heart sounds, additional sounds or murmurs were not audible. Abdominal distension and ascite was found. Other system examinations were unremarkable. 
In biochemical evaluation; complete blood count was normal; aspartate transaminase $284 \mathrm{IU} / \mathrm{l}$, alanine aminotransferase 452 IU/l, gamma glutamyl transpeptidase $128 \mathrm{IU} / \mathrm{l}$, total protein 6.2 $\mathrm{g} / \mathrm{dl}$, and albumin $3.2 \mathrm{~g} / \mathrm{dl}$, and mild bilirubin elevation were found. Acute phase reactants were negative. Cardiothoracic ratio and pulmonary vascular markings were normal on chest X-ray. Electrocardiography showed bi-atrial dilatation. Echocardiography revealed normal left ventricular dimensions and systolic functions; both left and right atria were enlarged (Figure 1). Mitral inflow velocities varied significantly with respiration (mitral E flow velocity at inspiration $0.64 \mathrm{~m} / \mathrm{sec}$, mitral E in expiration $1.02 \mathrm{~m} / \mathrm{sec}$ ). Tissue Doppler mitral E' was normal $(0.98 \mathrm{~m} / \mathrm{sec})$. Constrictive and restrictive cardiomyopathy was considered in differential diagnosis. Thoracic computed tomography (CT) and diagnostic catheter angiography were scheduled. Pericardial thickness had increased $2.8 \mathrm{~mm}$ and foci of pericardial calcifications were present on thorax CT (Figure 2). Catheter angiography revealed increased right atrial and pulmonary wedge pressures with equalization of end diastolic pressures. The following measurements were obtained: pulmonary artery wedge $18 \mathrm{mmHg}$, pulmonary artery pressure $30 / 20 \mathrm{mmHg}$, right ventricular pressure $31 / 17 \mathrm{mmHg}$, right atrial pressure $16 \mathrm{mmHg}$, left ventricular pressure $80 / 20 \mathrm{mmHg}$, and aortic pressure $79 / 50 \mathrm{mmHg}$. Pericardiectomy was performed. Histologic examination of the pericardial tissue was compatible with chronic fibrinous pericarditis. Since, tuberculosis is one of the leading causes of CP, and prevelant in Turkey, Quantiferon test was performed and resulted negative. The patient's clinical state improved dramatically after surgery, and the findings of right heart failure resolved entirely at two-year follow-up. Liver graft functions returned to normal levels, and the transplanted graft was saved. Thus, re-transplantation was not performed. The child had no signs of liver or heart failure. A written consent was obtained from the patient's parents for the publication of this case report and any accompanying images.

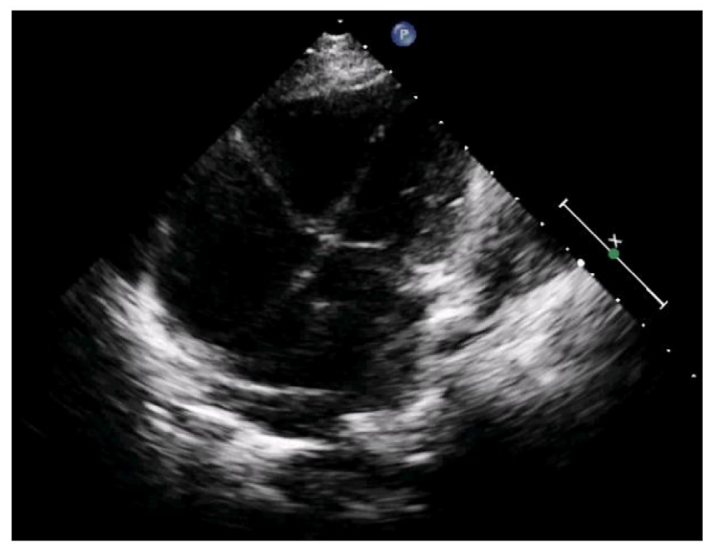

Figure 1. Biatrial dilatation in 4-chamber view on 2 D echocardiograpy.

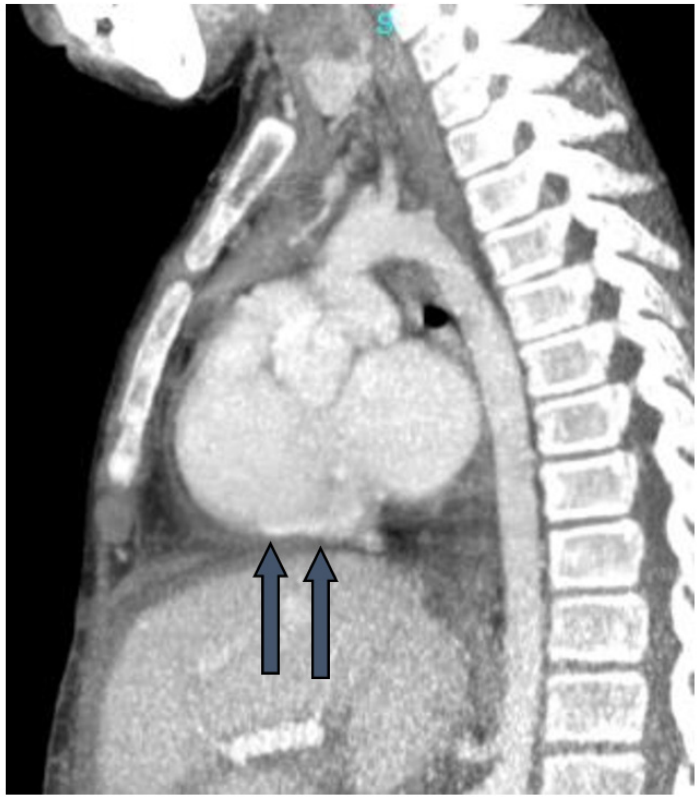

Figure 2. Computed tomography revealed pericardial thickening and microcalcifications.

\section{DISCUSSION}

Constrictive pericarditis is a clinical syndrome occurring due to chronic inflammation causing fibrotic thickening, calcification and adhesion of the pericardial layers which causes impaired diastolic filling of the ventricles. Tuberculosis, radiation, uremia, connective tissue diseases, trauma, heart surgery, and malignancy may be found as the underlying etiology; however, the majority of cases are idiopathic [4,5]. Liver transplantation related constrictive pericarditis is reported in only one adult patient previously [6]. Constrictive pericarditis may present with sign and symptoms of chronic renal disease, hepatomegaly and ascite may develop due to right heart failure. Other causes of graft dysfunction following liver transplantation may be bacterial or fungal peritonitis, obstruction of hepatic and portal veins or graft rejection [3,7-9]. On the other hand, several adult cases with CP has been reported following renal transplantation resulting graft dysfunction [10]. Absence of infectious causes and venous obstruction and dramatic improvement after pericardiectomy suggests constrictive pericarditis as the cause of liver dysfunction in our patient. It is difficult to understand if the initial liver dysfunction leading to liver transplantation was also related to constrictive pericarditis or constrictive pericarditis has developed as a consequence of the first transplantation in our patient retropectively since the cardiac evaluation before the first operation has been performed in another center and data at hand is inadequate. However, to our knowledge this is the first pediatric case with CP following liver transplantation.

As the pericardium becomes rigid in $\mathrm{CP}$, ventricular filling is restricted, cardiac compliance decreases, systemic, hepatic, and pulmonary venous congestion occurs due to diastolic 
dysfunction. Compromised blood flow results in equalization of diastolic pressures in the right and left ventricles and the pressure in the splanchnic system raises [2]. In the subsequent stages, hepatic congestion may progress to fibrosis and then to cirrhosis, resulting in organ loss.

The clinical findings of CP can be highly variable, usually patients with CP present with weakness, easy fatigue, dyspnea, cough, weight loss, and chest pain. Jugular vein distension, ascites, hepatosplenomegaly, pretibial edema, muffled heart sounds and pericardial knock may be found in physical examination [11]. Our patient also had early fatigue and shortness of breath, and physical examination revealed the abdominal distension and pretibial edema.

Transthoracic echocardiography is helpful in the differential diagnosis of right heart failure. Decreased pericardial movement and increased pericardial echogenicity can be seen on echocardiography in patients with CP. Enlargement of the atria and vena cava superior and inferior, and attenuation of respiratory movements of the vena cava inferior may be detected [2,12]. Enlargement of both atria was present on echocardiography in our case which may suggest either $\mathrm{CP}$ or restrictive cardiomyopathy (RCMP). Doppler echocardiography is a useful method for differentiating CP and RCMP. A marked change occurs in $\mathrm{CP}$, with respiration in the early ventricular diastolic volume rates, with tricuspid velocity increasing with inspiration and mitral flow velocity decreasing. A change exceeding $25 \%$ in mitral flow velocity with respiration is suggestive of CP. This finding is extremely uncommon in RKMP [12]. A marked change in mitral inflow was observed with respiration in the present case.

Differential diagnosis of CP and RCMP is a challenge in cardiology, which was also the case in our patient since some infiltrative diseases may cause both liver disease and restrictive cardiomyopathy. Diastolic dysfunction causes similar clinical and hemodynamic features in CP and RCMP. Atrial dilatation is more marked and ventricular systolic dysfunction may be present in RCMP. Presence of pericardial thickening, septal bouncing, prominent respiratory velocity changes in diastole suggests CP. Tissue Doppler velocity of lateral mitral annulus in diastole is normal or increased in CP while decreased in RCMP. $\mathrm{E} / \mathrm{E}$ ' decreases in CP.

Computed tomography and magnetic resonance imaging are useful non-invasive diagnostic tools with excellent sensitivity $(88 \%)$ and specificity (100\%) permitting direct visualization of pericardial thickening and calcifications [13]. Thoracal CT revealed pericardial thickening and calcifications in our patient.

Catheter angiography demonstrated elevated and equalized enddiastolic pressures of both ventricles and elevated pulmonary artery wedge pressure in our case that also supported the diagnosis of CP.

Pericardiectomy is the treatment of choice for symptomatic and severe $\mathrm{CP}$, with surgical mortality rates of $6 \%$ and sevenyear survival of approximately $88 \%$ [14]. Our case was referred for surgery and pericardiectomy and pericardial biopsy was performed at the same time. Diagnosis was confirmed when chronic fibrinous pericarditis was reported in histologic examination of pericardial tissue. Clinical condition of our patient improved dramatically after surgery, and the liver graft was saved.

Constrictive pericarditis must be considered and searched for in patients with symptoms of liver failure either before and after liver transplantation since it is a treatable condition by surgery. Our case is the first reported case of CP following LT.

Conflict of interest: The authors have no conflicts of interest to declare.

Funding: No financial support was received for this study. The authors have no financial or proprietary interest in any material or method mentioned.

Authors' Contributions: NC: Coordinated and supervised data collection, drafted the initial manuscript, reviewed and revised the manuscript, BSC: Conceptualized and designed the case, coordinated and supervised data collection, EE, KA and ET : Had active roles in the diagnosis and treatment of the patients, FA : Critically reviewed and revised the manuscript. All authors read and approved the final version of the article.

\section{REFERENCES}

[1] Nishimura RA. Constrictive pericarditis in the modern era: a diagnostic dilemma. Heart 2001;86:619-23. doi: 10.1136/ heart.86.6.619.

[2] Bergman M, Vitrai J, Salman H. Constrictive pericarditis: A reminder of a not so rare disease. Eur J Intern Med 2006; 17 : 457-64. doi: 10.1016/j.ejim.2006.07.006.

[3] Gotthardt DN, Weiss KH, Rathenberg V, Schemmer P, Stremmel W, Sauer P. Persistent ascites after liver transplantation: etiology, treatment and impact on survival. Ann Transplant 2013; 18: 378-83. doi: 10.12659/ AOT.883982.

[4] Szabó G, Schmack B, Bulut C, et al. Constrictive pericarditis: risks, aetiologies and outcomes after total pericardiectomy: 24 years of experience. Eur J Cardiothorac Surg 2013;44:1023-8. doi: $10.1093 /$ ejcts/ezt138.

[5] Myers RB, Spodick DH. Constrictive pericarditis: clinical and pathophysiologic characteristics. Am Heart J 1999;138:21932. doi: 10.1016/s0002-8703(99)70105-5.

[6] Bezjak M, Kocman B, Jadrijević S, et.al. Constrictive pericarditis as a cause of refractory ascites after liver transplantation: A case report. World J Clin Cases 2019;7: 3266-70. doi: 10.12998/wjcc.v7.i20.3266.

[7] Runyon BA. AASLD Practice Guidelines Committee. Management of adult patients with ascites due to cirrhosis: an update. Hepatology 2009; 49: 2087-107. doi: 10.1002/ hep.22853.

[8] Lominadze Z, Kia L, Shah S, Parekh K, Levitsky J. Constrictive pericarditis as a cause of refractory ascites. ACG Case Rep J 2015; 2: 175-7. doi: 10.14309/crj.2015.46.

[9] Barry M, Al-Muhaidb S, Fathala A. A case report of constrictive pericarditis: a forgotten cause of refractory 
ascites. Radiol Case Rep 2020;15:2565-8. doi: 10.1016/j. radcr.2020.09.053.

[10] Celebi ZK, Keven K, Sengul S, et al. Constrictive pericarditis after renal transplantation: three case reports. Transplant Proc 2013; 45: 953-5. doi: 10.1016/j.transproceed.2013.02.060.

[11] Ling LH, Oh JK, Schaff HV, et al. Constrictive pericarditis in the modern era: evolving clinical spectrum and impact on outcome after pericardiectomy. Circulation 1999;100:1380-6. doi: 10.1161/01.cir.100.13.1380.

[12] Welch T.D, Ling L.H, Espinosa R.E, et al. Echocardiographic diagnosis of constrictive pericarditis Mayo Clinic criteria.
Circulation: Cardiovascular Imaging 2014; 7:3 526-34. doi: 10.1161/CIRCIMAGING.113.001613.

[13] Howard JP, Jones D, Mills P, Marley R, Wragg A. Recurrent ascites due to constrictive pericarditis. Frontline Gastroenterol 2012; 3: 233-7. doi: 10.1136/flgastro-2012-100173.

[14] Chowdhury UK, Subramaniam GK, Kumar AS, et al. Pericardiectomy for constrictive pericarditis: a clinical, echocardiographic, and hemodynamic evaluation of two surgical techniques. Ann Thorac Surg 2006; 81: 522-9. doi: 10.1016/j.athoracsur.2005.08.009. 